\title{
A COMPLETE FOS APPROACH FOR INDOOR CROWDSOURCED MAPPING: CASE STUDY ON SAPIENZA UNIVERSITY OF ROME FACULTIES
}

\author{
A. Mascitelli ${ }^{1}$, M. Ravanelli ${ }^{1 *}$ S. Mattoccia ${ }^{2}$, C. Berardocco ${ }^{1}$, A. Mazzoni ${ }^{1}$ \\ ${ }^{1}$ Geodesy and Geomatics Division, DICEA - Sapienza University of Rome, Rome, Italy \\ $<$ alessandra.mascitelli, michela.ravanelli, augusto.mazzoni >@uniroma1.it \\ ${ }^{2}$ Territory's Management and Enhancement Master Degree - Sapienza University of Rome, Rome, Italy \\ stefano.mtci@gmail.com \\ berardocco.1719095@studenti.uniroma1.it
}

Commission IV

KEY WORDS: Crowdsourced Indoor Mapping, VGI, FOS, Geo-crowdsourcing, OSM Community

\begin{abstract}
:
Indoor mapping is an essential process in several applications such as the visualization of space and its utilization, security and resource planning, emergency planning and location-based alerts and, last but not least, indoor navigation. In this work, a completely free and open-source (FOS) approach to map indoor environments, and to navigate through them, is presented. Our tests were carried out within Sapienza University of Rome public buildings; in detail, Letters and Philosophy faculty and Engineering faculty indoor environments were mapped. To reach this goal, only open source software such as Quantum GIS (QGIS) and open-source platforms like Open Street Map (OSM) and its indoor viewer, Open Level Up (OLU) were adopted. A database of indoor environments of the two faculties, completely compatible with OLU, was created through QGIS. In this way, a public territorial information system of classrooms, offices and laboratories is accessible to everyone who can, hence, add or modify the information, following the principle of crowdsourcing and of Volunteered Geographic Information (VGI). The developed procedure is now standard and its outputs accepted by the OSM community. Hence, the long-term developments of this project are the proposal for the volunteered and cooperative indoor mapping and design of strategic buildings and infrastructures (hospitals, schools, public offices, shopping centers, stations, airports etc.), starting from the available information (indoor layouts) and knowledge acquired through experience of people who normally work inside them and/or visit them frequently. In this context it is possible to state that the development of VGI for internal maps for strategic buildings, infrastructures and denied GNSS environments, not only supports and improves internal and external navigation without interruption, but can also have a significant positive impact on security and emergency management.
\end{abstract}

\section{INTRODUCTION}

Indoor mapping is an essential process in several applications such as the visualization of space and its utilization, security, emergency and resource planning and location-based alerts and, last but not least, indoor navigation (Sonnessa et al., 2020). For these reasons, nowadays companies and industries are investing in indoor mapping and positioning: Google (GOOGLE, 2020) and ESRI (ESRI, 2020) are just some of them, but it is important to highlight that these solutions are not open source. The present work is inserted in this background. The particular aim is to develop an interactive tool based on open source software; the project can be thus inserted in the wider context of online cartography. In particular, the tool developed is based on OpenStreetMap (Open Street Map, 2020), an open source collaborative project aimed at creating free content maps of the world. Generally, the data for the creation of the maps are obtained by volunteers who carry out surveys on the territory sometimes with GPS units capable of recording the route, sometimes with the help of smartphones, cameras, voice recorders, laptops or even just with paper. The information collected is sent via computer, or through some applications on smartphones, to the database in vector format. OpenStreetMap is, therefore, a geographical database and a clear example of VGI (Voluntereed Geographic Information). The VGI is the collective development of a project aimed at collecting geospatial information from numerous people outside the entity that conceived the project and who actively participate in it.

\footnotetext{
${ }^{*}$ Corresponding author
}

The people who collaborate do so voluntarily, responding to an invitation to collaborate. This model of project implementation is generally made possible by the Internet; the nature of the projects can be very different, not necessarily in the geospatial field (just think of Wikipedia, written by its readers). The use of this resource is of fundamental importance not only in ordinary situations, but also during extreme events. The case of the earthquake in Haiti, for example, has shown how territorial information systems can represent a fundamental tools through which individuals are able to make a tangible difference in the work of relief agencies (Zook et al., 2010). The open source community can be thus a link between ideas and products. The product generated through OpenStreetMap is undoubtedly a map but, unlike classic maps, you can access the data "below" that have helped to enrich it. There are therefore many advantages: it is easy to map new objects or improve existing ones; the availability of specialized maps (for cyclists, hikers, etc...); the possibility to download data, filter and analyze them; the possibility to insert new data, such as Open Data of regions and municipalities, that can be used as a background layer for further views and even devise new creative or unexpected uses.

In this context, however, the need is to have the possibility to map not only external environments, but also internal ones, so it is necessary to rely on another tool: OpenLevelUp. Openlevelup.net is a web map that can show the interior of buildings, level by level, interactively. This tool relies on the servers of OpenStreetMap and is extremely useful for viewing indoor data. OpenLevelUp is able to describe the various buildings with appropriate styles, adding a lot of information, 
specifically related to indoor themes (Open Level Up, 2020).

At present there are still very few indoor environments mapped through this methodology, which underlines its novelty. Some cases, however, can be found in some stations, commercial buildings and universities, especially in France, where the platform was designed (Pavie, 2020), while in Italy the indoor mapping has appeared only in recent years. The first Italian project was the one applied to the Faculty of Engineering of the Sapienza University of Rome. If, therefore, the activity began as an application of a best practice to a context that had not yet seen its potential application, on the other hand, the implementation has concealed a series of complications related to the complexity of the plans. The Faculty of Engineering of the Sapienza University of Rome is characterized by the coexistence of historical buildings and buildings of more recent construction. This level of complexity required a considerable effort to make these building layouts compatible with Open Level Up platform. The paper is divided in two main sections, the design process paragraph and the results paragraph. The first concerns the preliminary procedure for the management of the material and is divided into four sub-sections: Layout management, Georeferencing, Tagging and Upload on JOSM. The second one includes an overview of the outputs and a sub-section dedicated to the test application of Indoor mapping for participatory planning. In the end a conclusion paragraph, which includes also prospects, is reported.

\section{DESIGN PROCESS}

The project aims at indoor navigation in two faculties of Sapienza University of Rome: the faculty of Civil and Industrial Engineering and the faculty of Letters and Philosophy. Obviously, in these indoor environments it is not available the GNSS coverage.

The design process can be divided into three main phases.

The first concerned the retrieval of the layouts and their import and vectorization in a GIS (Geographic Information System) software, assigning them the chosen reference frame and constructing the respective geometries. The second phase concerned the description of the layouts with the insertion and enrichment of a series of information collected through field surveys, and performing a roto-translation with scale factor. Finally the layouts, previously framed in the respective reference frame and saved in a suitable format, were imported into the Open Street Map remote editor, and then uploaded into the platform and made available online to all users.

\subsection{Layout management}

The layouts of the two faculties were provided from Sapienza University of Rome in *.dxf format. The layouts were converted into *.shp files (ESRI shape file), since it is the only format compatible with OSM. To this aim, Quantum Gis software (QGIS) was employed: an open source desktop application with a free license, maintained and updated in its versions by a group of volunteer developers (Quantum GIS, 2020). The layouts were initially lacking essential information such as the reference system and orientation. The layouts were oriented in the WGS84 reference frame, using UTM zone $33 \mathrm{~N}$ projection (EPSG: 32633). Furthermore, the vectors with polygonal geometry were loaded, since they are the only ones useful for our goal. Nevertheless, the layouts are still too complex and too heavy to be loaded on OSM. It was, therefore, necessary to simplify them through QGIS. A new polygonal layer was created that allowed to trace the perimeters of the environments of each floor by superimposing the latter on the existing layers (which were then obscured). In this way it was possible to reconstruct the layout of the environments in the plans, for each level, creating much simpler floor plans than the original ones. Figure 1 shows the difference of the original layout (top panel a) ) from the simplified one (bottom panel b) ).

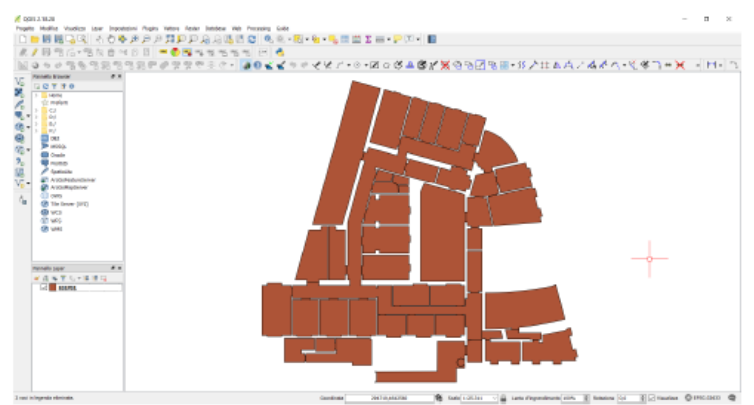

(a)

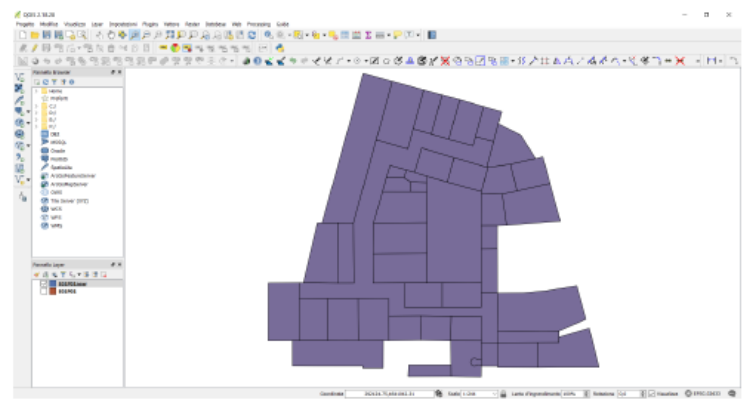

(b)

Figure 1. (a) The top panel shows the original layout. (b) the bottom panel depicts the simplified layout.

\subsection{Georeferencing}

Georeferencing is the attribution of map or aerial photo image to a system of geographic or map projection coordinates, linked to a selected reference frame, here (WGS84, projection UTM $33 \mathrm{~N}$ ). The assignment of the coordinates to the points of an image takes place using check points (Ground Control Points) identified on a topographic map or on a georeferenced image. A single control point for each building was found and the coordinates were assigned to the corresponding point on the layouts. The layouts were, then, reduced, moved and rotated. In this way they are perfectly superimposed to form the various levels, making them tied with the Open Street Map map. Specifically, QGIS Quantum aided Design (QAD) plugin was employed: it allows to perform operations usually performed in the CAD design field in a GIS environment.

First of all, it was necessary to scale the layouts, with a QAD function. This function allows you to quickly and easily reduce the size of an object; after selecting the layout, it is enough to specify only one point ("Base point") and the scale factor. The chosen scale factor is equal to 0.01 , which corresponds to the scale of the layouts provided by the University (1:100).

Subsequently, the layouts have to be translated and rotated. The translation takes place via the "move" command, a special QAD function. Before using this command, it is necessary to identify a control point whose coordinates will be assigned to the corresponding point on the layout. To achieve this goal, Open Street Map was loaded in QGIS and WGS84-UTM zone $33 \mathrm{~N}$ coordinates were applied. After identifying the coordinates of the Ground Control Point the layout was translated through the "move" command. In particular, it is necessary to specify 
the displacement base point, which is the point on the layout corresponding to the control point of the map, to which the coordinates previously identified will be assigned. Figure 2 represents the layout at the end of the translation step.

Eventually the layouts have to be rotated in order to make them

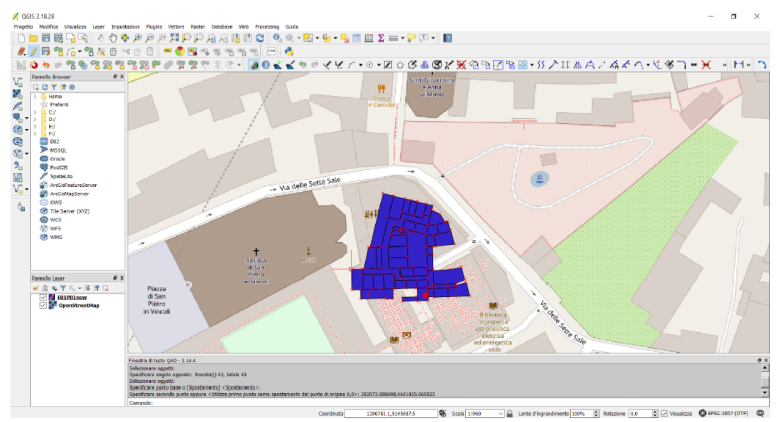

Figure 2. The translated layout of the first floor of faculty of Civil and Industrial Engineering building

coincident with the cartographic representation in Open Street Map. To perform this last step, the QAD "rotate" button was used. Also in this case a base point is identified and the rotation angle is specified. The value of the rotation angle was identified by trial and error up to obtain a perfect coincidence between the layout and the perimeter of the building on the OSM map. Figure 3 shows the QAD button tool used in this step and the layout perfectly coincident with the OSM map beyond.

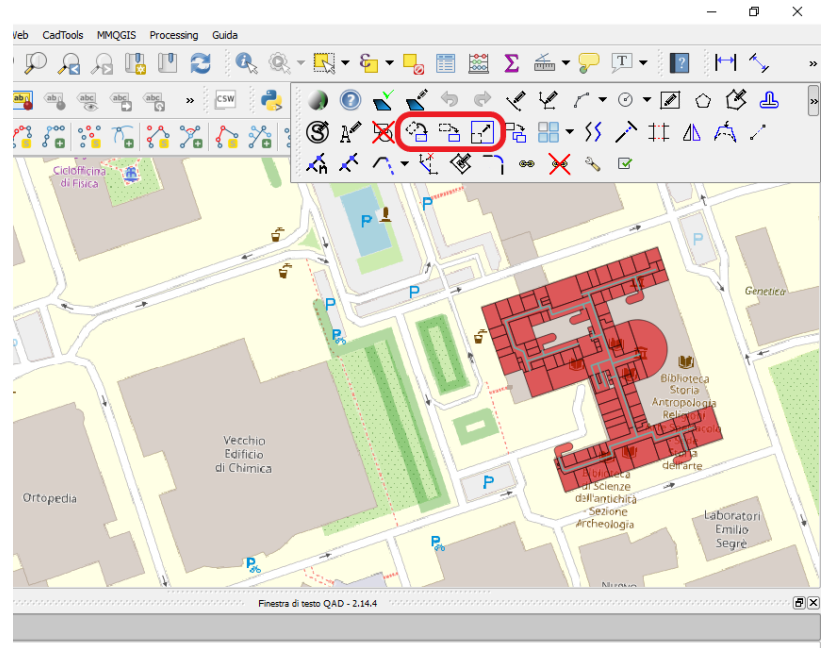

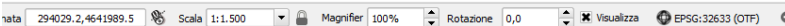

Figure 3. QAD button tool used in this step and the layout perfectly coincident with the OSM map below

\subsection{Tagging}

At this step, it is necessary to enter the information suitable for indoor navigation. The information gathering was obtained through several surveys, which clarified the use of the various rooms within the building of the two faculties.

In particular, keys and values respecting the nomenclature of Open Level Up were employed. In this way, the combination of tags will result to an icon to be shown on Open Level Up for each "tagged" environment. To this aim, QGIS attribute table was used. The attribute table for each vector was opened and various tag columns were added. For each selected element of the geometry, the appropriate information was added for each tag (each column). In this phase, it was possible to add a link to the web page in correspondence with the "notes" key, aimed at involving users in the planning phase of certain environments. Figure 4 shows the attribute table of the first floor of one building of the Civil and Industrial faculty.

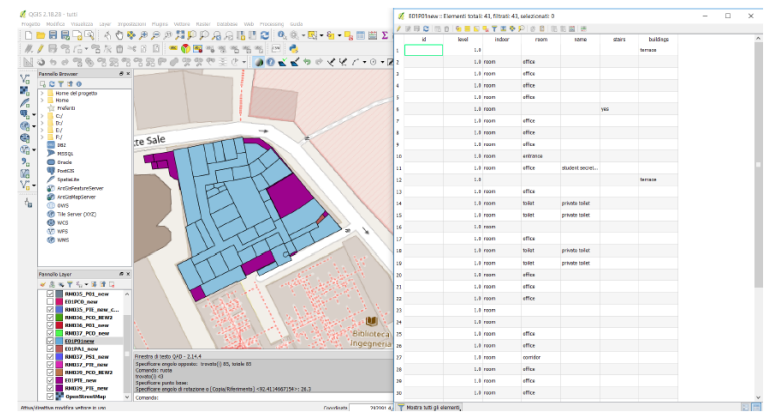

Figure 4. Attribute table of the first floor of of one building of the Civil and Industrial Engegnering faculty.

\subsection{Upload on JOSM}

In the last phase, the layouts in *.shp file format were loaded on the Java Open Street Map (JOSM) platform. JOSM is an OpenStreetMap data editing software, having the advantage to work remotely, performing tests and modifications off-line (Java Open Street Map, 2020). In JOSM, it is possible to download the area of interest map from Open Street Map. The layouts are, hence, positioned in area of interest thanks to the previous georeferencing process. Figure 5 shows the visualization of the layout within the JOSM environment. Eventually, the layouts level by level were so loaded on Open Street Map and, hence, can be visualized on Open Level Up platform. According to the aims of indoor navigation, additional information were added: the paths, information about the passage from one venue to another. Path creation was done using the JOSM Editor. Specifically, paths are lines tagged with specific keys.

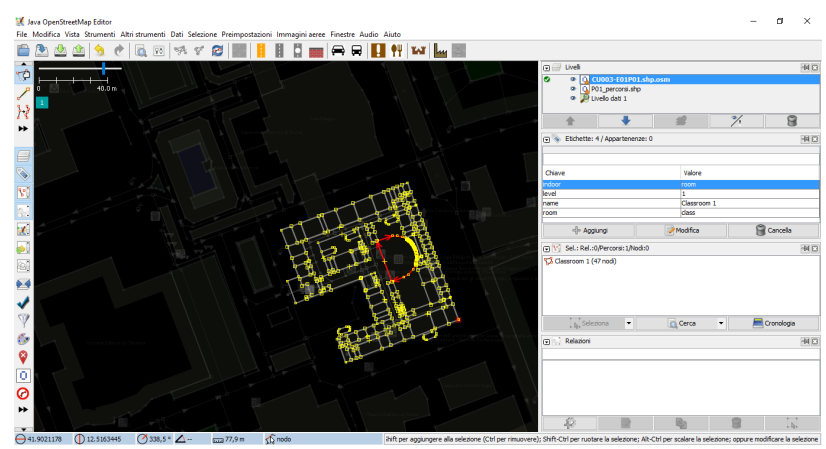

Figure 5. Visualization of the first floor of the faculty of Letters and Philosophy within the JOSM environment

\section{RESULTS}

The final results of this work is completely available on Open Level Up platform (Open Level Up, 2019a), (Open Level Up, 2019b). In particular, Figure 6 and Figure 7 represent respectively the ground floor of Geography faculty and the first floor of Engineering faculty. These Figures give an idea of the final 
results of the indoor mapping. It is possible to navigate through levels by clicking the "Up" and "Down" arrows on the top of the left sidebar.

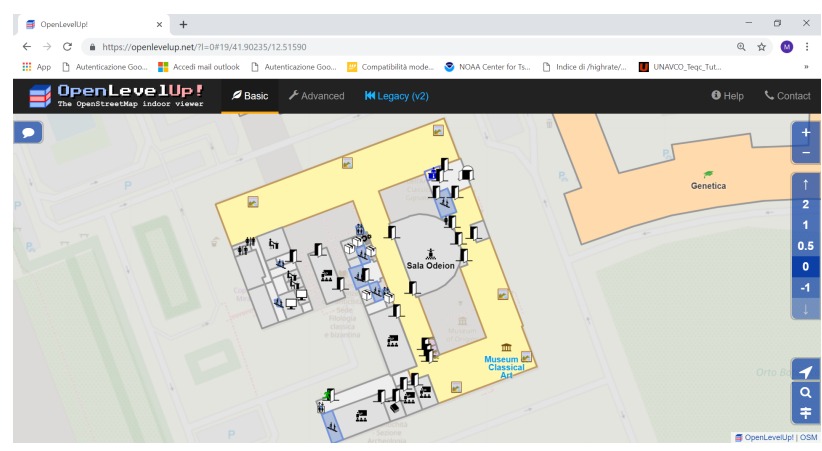

Figure 6. Open Level Up indoor map of the faculty of Letters and Philosophy

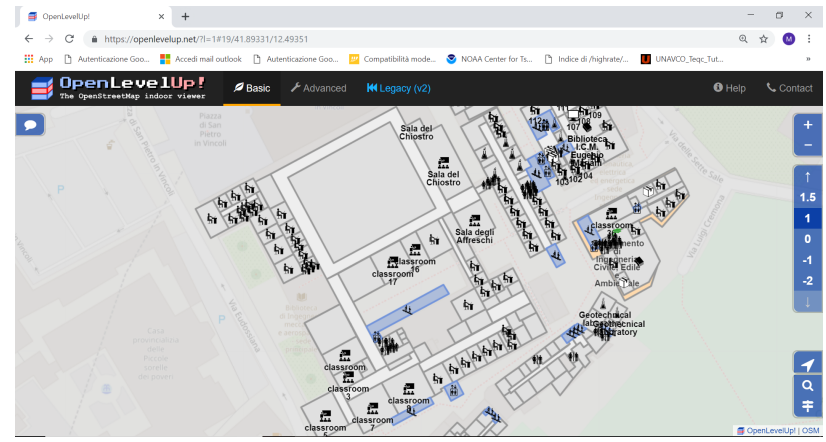

Figure 7. Open Level Up indoor map of the faculty of Civil and Industrial Engineering

Thanks to the indoor navigation tool, it is possible to compute paths to go from a level to another. Anyway, this feature is not yet implemented in the new version of Open Level Up, but just in the Legacy one. Figure 8 represents an example of navigation within indoor environments.

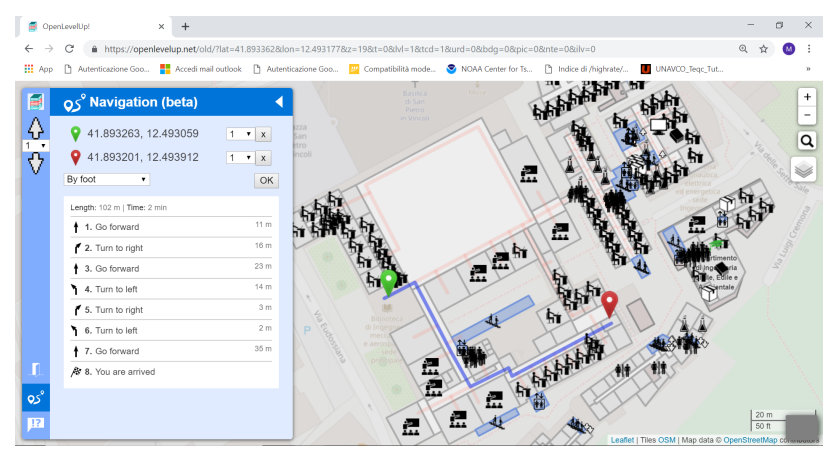

Figure 8. Open Level Up indoor map of the faculty of Civil and Industrial Engineering

\subsection{Indoor mapping for participatory planning}

Another aspect of considerable interest was to use the online platform OpenStreetMap as a bridge for the use of participatory planning.

The transformations in the organization and functioning of the territories are determined by both local and global factors; the former contribute to giving material body to the territories, while the latter are fundamental for the identification of developments. it is in this context that the idea of participation takes root, with the intention of inducing the local potential to escape and evolve, in order to be integrated into a global context. The intention is to encourage constructive debate and confrontation, because only with fruitful communication between stakeholders becomes possible to achieve a shared goal.

In the study of the (Lefebvre, 1974), the space is split into the three components of: Perceived Space (action), Conceived Space (thought), Living space (experience). In this perspective, space is analyzed and described as the sum of three moments. The first is the space of spatial practices, understood as a space in which people carry out their tasks; the second is the so-called "experts' space" and concerns the representations of space coming from a form of scientific knowledge; the third and last one is instead the space of the representations, it is the space experienced through the images and symbols that accompany it, it is the dominated and suffered space that the imagination tries to possess and modify. Taking into account this theory, we have tried to find out in practice what Lefebvre defines as "Social production of space", paying attention to how the territory covered by this study is perceived, conceived and imagined by the people who inhabit it daily. In this specific case, a link was generated to a web page dedicated to the project of the Museum of Geography, at the faculty of Letters and Philosophy of Sapienza University of Rome (Museum web page, 2019). In the page, it is possible to view the current project and send comments, with the aim of contributing actively and in the planning stage to the proposal. This link was loaded in OpenLevelUp in the notes related to the internal environment object of the proposal. This will make it possible to have a clear view of the current design and the proposed changes.

\section{CONCLUSION AND FUTURE WORK}

In this work, a completely free and open-source (FOS) approach to map indoor environments, and to navigate through them, is presented. Our tests were carried out within Sapienza University of Rome public buildings; in detail, the faculty of Civil and Industrial Engineering and the faculty of Letters and Philosophy were mapped. A database of their indoor environments is completely available on OLU (Open Level Up, 2019a), (Open Level Up, 2019b). In this way, a public territorial information system of classrooms, offices and laboratories is accessible to everyone who can, hence, add or modify the information, following the principle of crowdsourcing and of Volunteered Geographic Information (VGI).

Furthermore, this procedure is repeatable from all the users, since only free and open source (FOS) platforms and software were employed.

The automatization of the layout simplification/generation may represent a future development of our work. In this background, the work of (Capocchiano et al., 2017), (Capocchiano, Ravanelli, 2019), focused on the automatic extraction of layouts from the $3 \mathrm{D}$ models of rooms acquired by means of range cameras and laser scanners, can represent an important improvement to this methodology, since range camera and laser scanner technologies are increasingly evolving. This outlook may be very promising, since this technology can be potentially used by non-expert users when the building geometry is reconstructed, to collect crowdsourced indoor information. This tool highlights the considerable potential in the phase of survey of environments by regular users. Indeed, the VGI is fundamental in the step of inspection and recording of existing information and it is also useful in the planning process, when the empirical knowledge of the habitual users can be of considerable support to the planning 
of the environments.

In conclusion, the long-term developments of this project are the proposal for the volunteered and cooperative indoor mapping of strategic buildings and infrastructures (hospitals, schools, public offices, shopping centers, stations, airports etc). In this way, it could be employed the available information (indoor layouts) and knowledge acquired through experience of people who normally work inside them and/or visit them frequently. Moreover, the proposed procedure is in principle one-to-one applicable also to outdoor environments where, due to relevant obstructions, GNSS signals are scarcely or not at all available (e.g. historical centers with narrow streets). In the end, within a 10-years time scale, the VGI development for indoor maps and navigation for strategic buildings, infrastructures, and GNSS denied environments, cannot only support and enhance the seamless outdoor-indoor navigation, but also can have a considerable positive impact on the safety and security and emergency interventions management.

\section{REFERENCES}

Capocchiano, F., Ravanelli, R., 2019. AN ORIGINAL ALGORITHM FOR BIM GENERATION FROM INDOOR SURVEY POINT CLOUDS. The International Archives of the Photogrammetry, Remote Sensing and Spatial Information Sciences, XLII-2/W13, 769-776. doi: 10.5194/isprs-archives-XLII-2-W13-769-2019.

Capocchiano, F., Ravanelli, R., Crespi, M., 2017. A TOOL FOR CROWDSOURCED BUILDING INFORMATION MODELING THROUGH LOW-COST RANGE CAMERA: PRELIMINARY DEMONSTRATION AND POTENTIAL. The International Archives of the Photogrammetry, Remote Sensing and Spatial Information Sciences, XLII-2/W8, 75-81. doi: 10.5194/isprs-archives-XLII-2-W8-75-2017.

ESRI, $2020 . \quad$ https://www.esri. com/esri-news/releases/18-3qtr/

esri-announces-new-indoor-mapping-product.

GOOGLE, 2020. www.google.com/maps/about/partners/ indoormaps/.

Java Open Street Map, 2020. https ://josm.openstreetmap. $\mathrm{de} /$.

Lefebvre, H., 1974. La production de l'espace. Anthropos, Paris.

Museum web page, 2019. http://www.kuaternion.com/ ppmuseo.html.

Open Level Up, 2019a. https://openlevelup.net/?1=0\# 19/41.90235/12.51590.

Open Level Up, 2019b. https://openlevelup.net/?1=1\# 19/41.89331/12.49351.

Open Level Up, 2020. https://wiki.openstreetmap.org/ wiki/OpenLevelUp.

Open Street Map, 2020. https : //wiki .openstreetmap.org/ wiki/Main_Page.

Pavie, 2020. https://pavie.info/cas-usages/.

Quantum GIS, 2020. https://qgis.org/it/site/.

Sonnessa, A., Saponaro, M., Alfio, V. S., Capolupo, A., Turso, A., Tarantino, E., 2020. Indoor positioning methods -A short review and first tests using a robotic platform for tunnel monitoring. ICCSA2020. Under submission.
Zook, M., Graham, M., Shelton, T., Gorman, S., 2010. Volunteered geographic information and crowdsourcing disaster relief: a case study of the Haitian earthquake. World Medical \& Health Policy, 2(2), 7-33. 